\title{
FOSTERING A LEARNING EXPERIENCE THAT IMPROVES KNOWLEDGE EXCHANGE BETWEEN ACADEMIA AND INDUSTRY
}

\author{
Laura FERRARELLO \\ Royal College of Art, United Kingdom
}

\begin{abstract}
$21^{\text {st }}$ Century technological advancements have developed a new kind of economy drawing from the capability of knowledge to create innovation and social growth. This has created new market opportunities but also destabilised jobs, those needing retraining and upskilling. Driven by the motivation of providing future generations with an attitude that is skilled to tackle and challenge the transformations society is currently undertaking this paper describes a project developed by the postgraduate researchers from the interdisciplinary Royal College of Art MRes programme; this explores an approach to education focussed on the human experience and attitude towards learning enabled by cross-discipline collaboration, debate, critical enquiry, peer-to-peer learning and creativity. The intention is to support the development of a lifelong learning culture that contributes to the production of a kind of knowledge that generates new market opportunities based on human skills and capabilities. By deploying public engagement as a method to coordinate and enhance the quality of knowledge exchange between research and innovation, this paper describes how education can be transformed in an environment that leverages creativity, collaboration, reflection, criticality and evaluation to foster knowledge for the knowledge economy. Through a cross discipline dialogue, which makes the experience of learning a multidimensional and multilateral entangled journey, this paper aims to draw attention on the value the human capital can offer to the $21^{\text {st }}$ economy in the development of a process of innovation that is negotiated and interdisciplinary.
\end{abstract}

Keywords: Interdisciplinarity, public engagement, knowledge exchange, dialogue, learning experience

\section{INTRODUCTION}

The advancements digital technologies like AI (Deep Learning, Machine Learning, Computer Vision) and robots have been developing in society are not only redesigning the infrastructure of our organizations and institutions but also the way human skills and capabilities could support social innovation. According to the McKinsey report on Future of Work [7] more than 160 million new jobs will be created by 2030 which will generate new opportunities but also new challenges for those in need of retraining and upskilling. The main factor that has contributed to the disruption and transformation of work is the increased number of tasks delivered by robots and AI. This has on one hand devaluated human applied skills, like dexterity and precision, and on the other one has increased the value of human knowledge to the extent of becoming the currency of the $21^{\text {st }}$ Century economy [9]. The knowledge economy job demand is no longer based on a $20^{\text {th }}$ Century skill-based workforce [9] but on people able to develop ideas and innovation with technology.

A job market mainly dominated by knowledge, i.e. the ability to learn, evaluate and criticise, and creativity has introduced new skills like interdisciplinary collaboration, entrepreneurship, lifelong learning, proactivity, risk taking and collaboration which enhance the human value in human-totechnology interactions [1] and foster new kinds of business models [9]. However, to develop these skills at an unprecedent scale it is crucial to design the social transformations that could mitigate disruption and guide people through the upskilling journey. Traditionally training and education have been recognised as the social institutional means able to help people come to terms and adapt to social and economic changes. However the knowledge society has been raising questions on the validity of the current model of education in particular regarding its approach to learning which should be an experience that doesn't only provide notions and skills but also an epistemological method that gives 
people the capability to acquire and develop knowledge across a lifetime. Helping people become lifelong learners needs an education model able to foster and promote a new culture and experience of learning through an environment that nurtures new skills applicable to real case scenarios [9]. In other words to design a welfare capable of responding appropriately to $21^{\text {st }}$ Century economic and technological transformations education needs to rethink the value it plays in society by shifting its model from a discrete to a lifetime distributed ecosystem mentoring people's professional life [7].

What is the strategy education should adopt and deploy to train an open-minded community of selflearners? What methods can foster one's capability to creatively engage with challenges, to learn from each other and to collaborate across disciplines and sectors to transfer and develop new knowledge?

A distributed educational ecosystem needs to address these questions to mentor a resilient attitude to change and an inclusive lifelong learning culture which both stimulate those intellectual and practical skills capable of informing future careers whilst in training. Learning methodologies that acknowledge the market needs can guide social transformations empowering people in the evolution and transformation of work by leveraging the human capabilities of critical enquiry, imagination, evaluation, self-reflection, observation and curiosity [2].

This is the context that the Royal College of Art (RCA) MRes addresses; by deploying the exploratory nature of research as an environment able to stimulate the epistemological conditions leading to lifelong learning the programme is structured around interdisciplinary and collaborative projects that use different research methods and practices. Being across the RCA Schools of Design, Architecture, Communication and Art \& Humanities the MRes aims to generate a kind of research that is not homed in a discipline but entangles 4+ different cultures, processes and methodologies. This cross-discipline environment intends to support an attitude to learning which acknowledges that knowledge is not a consolidated set of notions but a dynamic and multi-perspective environment that is shaped by creative and critical collaboration and dialogue across people of different disciplines and cultures.

To enable this, it is important to design the human experience of learning based on the abilities of listening, observing, evaluating and assessing information of different nature. The MRes has been exploring this aspect through a project, Make it Public, that is centred on the role and value of engaging people in research. The strategic role of public engagement in research is renowned; it is indeed a method capable of developing new policies, testing new products and technology, disseminating research, helping decision-making and/or informing the public through different forms of dialogue which vary from discipline to discipline [8]. Through different formats - e.g. interviews, workshops, performances, etc- public engagement can support the validation, testing and dissemination of in progress or completed research. Being aware of the disparity this method might create if not well handled (e.g. the power relationships between the public and the researcher) [8] public engagement is undoubtedly capable of structuring a multi-perspective and multi-discipline debate and process aimed at developing knowledge. The MRes Make it Public project explores the use of public engagement as a method to stimulate an unsiloed and proactive experience of learning that questions disciplinary knowledge through the critical and creative dialogue emerging from different modalities of researching. The integration of engagement in the research process generates knowledge through the experiential feedback loops postgraduate researchers exchange through reflection, discussion, evaluation and communication across disciplines. This process constructs an epistemological experience that produces knowledge from a collaborative working environment in which postgraduate researchers acknowledge the value they play in the group and in the field of study. Multi-disciplinary collaboration is therefore a method for growing a personal and collective resilience towards learning that can stimulate an open-minded culture open to cross disciplinary dialogue and discussion. Therefore, supporting entangled learning experiences [6] through public engagement might inform a disseminated model of education able to create new knowledge from the singularity of experiences emerging from the combination of personal and collective ones.

\section{METHODS: DESIGNING KNOWLEDGE EXCHANGE}

\subsection{Dynamics of interactions between education, research and innovation}

In a process aiming at knowledge transfer/exchange it is important to understand the difference between transmission and participation: the first focusses on the "from-to" whilst the latter on the "in-between" dynamics [10]. A model that visualises this difference is the Knowledge Triangle (KT) (Figure 1) which represents with a triangular form the dynamics of knowledge transfer (from-to) and exchange (inbetween) between three factors: Academic Research (content), Education (method) and Innovation 
(outcome) [10]. With the intention to identify what strategies can foster an innovation drawn upon an increased quality of the interactions between these factors the KT model visualises an ecosystem that outlines the key role of the dynamics of interactions between the three factors and the feedbacks emerging from the whole system in generating knowledge exchange. Therefore, the KT model evidence that knowledge results from the influence each key factor plays on the other two and the whole system. Quality can be therefore generated from an effective coordination of the dynamics of interactions [4].

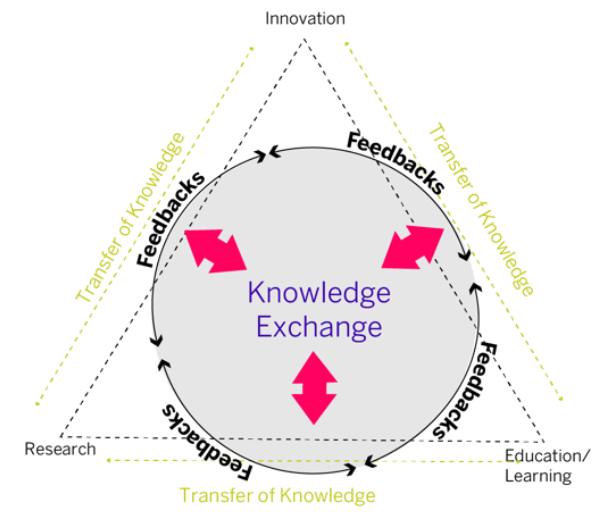

Figure 1. The Knowledge Triangle Diagram

The KT model indicates Education as method able to coordinate this system by defining it as an ecosystem where Innovation and Research coexist [11]; this status gives Education the ability to generate and provide the conditions for generating knowledge [3]. As described above knowledge exchange is developed upon the interactions of Research, Education, Innovation and the feedback loops between these. This implies that Education learns from the other two factors for positioning its coordinator role. The aspect that Education learns from Innovation is its entrepreneurship culture which proactive approach can stimulate qualitative dialogues between Innovation and Research by aligning strategies, goals and motivations [10]. However to be effective this dialogue needs particular skills of which interdisciplinarity and diversity score at the top of the list [4] for their capability of enhancing quality through the plethora of perspectives and understandings of knowledge that simultaneously interact to achieve a shared goal. A diverse dialogue can indeed stimulate creative, multidimensional and multidirectional exchange of knowledge between academia and industry which benefits both (Figure 2).

It then follows that an entrepreneurial, interdisciplinary and diverse model of education enables and enriches the infrastructure of knowledge exchange as it supports the different channels improving the quality of the transfer, i.e. peer to peer learning, creativity, self-reflections, discussion and evaluation. Knowledge exchange is therefore constructed through the interdisciplinary experience of transferring knowledge between academia and the industry which recognises the human skills of imagination and creativity [10] amongst others. The different understandings, approaches and interpretations different disciplines have of the same concept/problem develop a process of innovation which quality is enriched by collective creativity, i.e. a state of mind in which all our intelligences are working together involving seeing thinking and innovating [2]. Under these terms the development of new knowledge becomes an osmotic proactive process of exchange and transformation which is created, owned and acknowledged by any involved participant. 


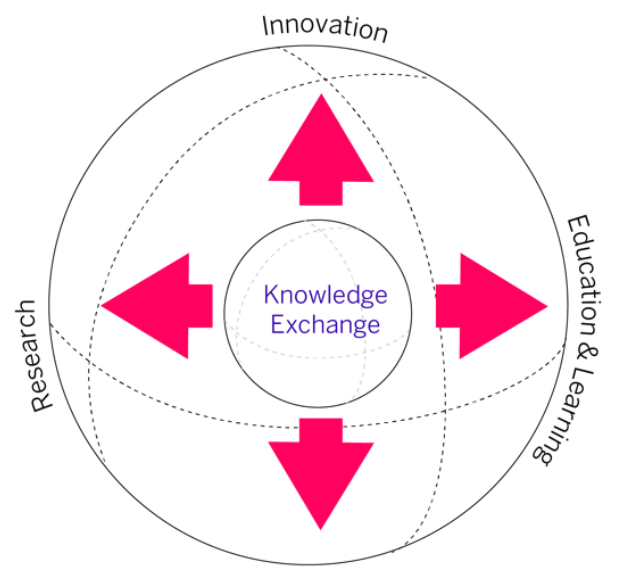

Figure 2. The influence of interdisciplinarity in the KT diagram

An entrepreneur culture in education can therefore construct a learning environment that guides social transformations through the dynamic, interdisciplinary, collaborative and creative dialogue between Research and Innovation. This draws attention to the key role the human experience and approach to learning and collaboration can play in generating qualitative interactions informing economic and social transformations.

\subsection{Public engagement as a method to foster knowledge exchange}

If a proactive entrepreneurship attitude in Education can generate the environment for knowledge exchange it is unknown what exact method can coordinate the dynamics of exchange. Given the positive influence the dynamic nature of human behaviour and creativity can provide to the development of new knowledge this method should support a multi-dimensional dialogue but also structure the dynamics of exchange. Designed upon the objective of fostering the exchange of knowledge between different backgrounds public engagement responds to this need; through different kinds of activities public engagement provides participants of the tools for developing knowledge through dialogue and debate [8]. As biunivocal process it offers how researchers can implement and test ideas and the public can access information and increase knowledge. Public engagement is therefore a method that manages coordination by supporting communication, dialogue and accessibility across cross-discipline participants. By doing so it empowers participants by giving them voice and space to express ideas and discuss different opinions [8]; therefore public engagement can leverage personal and collective creativity and imagination by making them visible to the group which helps the integration of disciplinary knowledge and expertise [5]. Under these terms public engagement is a suitable method that can coordinate the relationships and the dialogue between Research and Industry.

Based on these principles the RCA MRes Make it Public project has used public engagement to explore how to design an experience of learning that can stimulate an open minded and lifelong learning attitude. To achieve this the project has arranged postgraduate researchers from the RCA 4 schools in crossdisciplinary groups with analogue research directions to develop a festival in which selected topics become shared points of discussion and creative platforms of knowledge exchange. The 7 different topics (Balance, Body, Identity, Matter, Memory, Participation, Position) that the 7 groups selected use public engagement as a product of the festival and but also as method to coordinate the dynamics of discussion and debate that contribute to the process of developing the festival; the postgraduate researchers have prototyped their activities by debating and negotiating disciplinary concepts with their peers and external experts. Public engagement has guided the experience of developing the festival and working across disciplines by leveraging personal and collective critical and creative thinking, selfreflection, evaluation and capability to listen and develop knowledge with their peers. Indeed the 7 groups have developed different forms of creative engagement spanning from workshops, sound performance, publications, zines, interviews and webpages which ultimate goal is to visualise and negotiate a known and familiar context (the student's discipline) with unknown forms of collaborative work (the festival activity) that needs to be accessible to external experts and members of the public. For instance, the Position group, which group members are graphic designers, artists and architects, has developed an activity which builds from Brian Eno's Oblique Strategies cards. Through an Instagram 
account members of the public can work individually or in group in activities aiming at helping people of different theoretical positions to collaborate. The custom designed cards (Retool Box) are objects that support interdisciplinary work by respecting the diversity of the group. Generally any of the 7 projects have designed an experience of knowledge transfer that leverages any personal and collective participation as a heuristic approach to learning; members of the public are part of this experience through the work they contribute to produce. Under these terms the activities designed by the groups coordinate a creative space of reflection, communication and criticality but also stimulate new forms of learning and knowledge production. Learning in the Make it Public project is conceived as a creative and reflective journey developed by the postgraduate researchers in collaboration with the members of the public. Under these terms the 7 different activities act as boundary objects, i.e. as entities that enhance the capacity of an idea, theory or practice to translate across culturally defined boundaries [6]. These activities make learning a tangible experience developing knowledge that any participants understand and own under their terms.

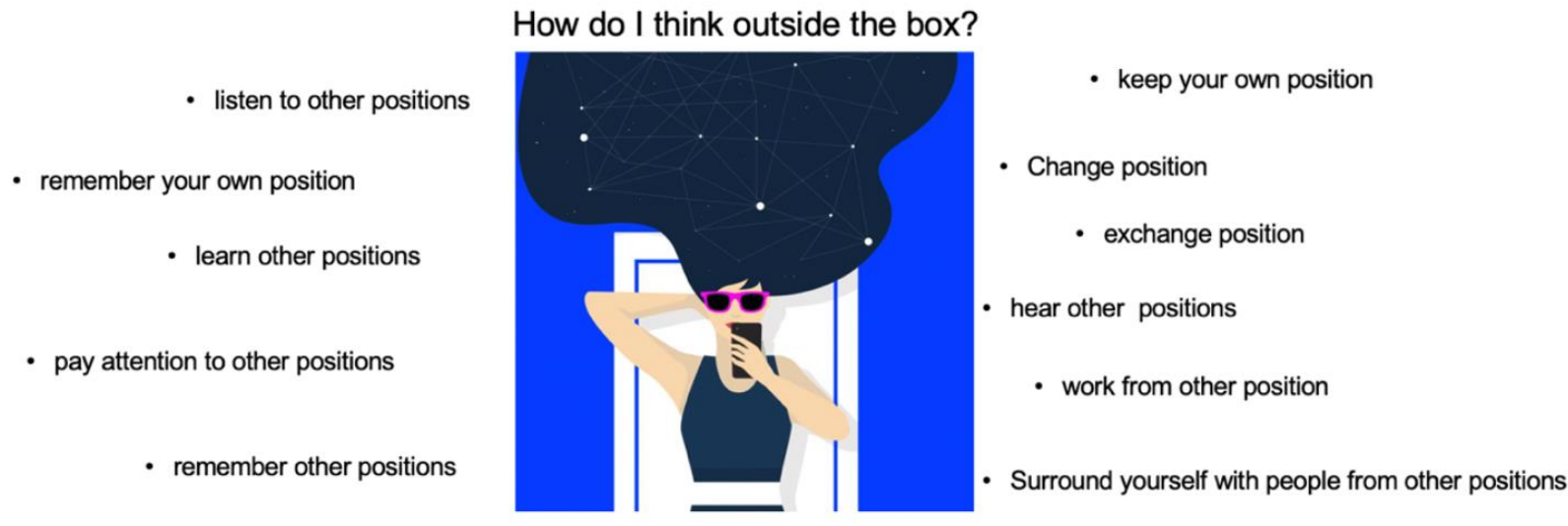

Figure 3. Principles developed by the Position group (@ Eleonora Antoniadou, Roxanne Bottomley, Sally Stenton, Marisa Ferreira, Melissa Lu, Yuzhen Cai, II Soon Moon)

\section{DISCUSSION AND CONCLUSION}

Designing an experience of collaborative, negotiated and interdisciplinary learning has been the goal the RCA MRes aimed to with the intention to stimulate an open minded and lifelong learning culture and also inform the development of a disseminated educational model able to support future generations in the adaptation to social transformations. Using public engagement as method that coordinates the relationships between Research and Innovation the MRes Make it Public project has explored a process which goal is to develop multi-directional creative dynamics with multi-disciplinary peers which leverage and encode reflection, criticality and evaluation to stimulate qualitative synergetic collaborations [5]. Using public engagement under these terms has stimulated a resilient and open minded attitude towards learning emerging from the contrast between an open-ended process and a limited way to express ideas via digital platforms only (this is because of the Covid-19 social distancing measures). The postgraduate researchers had been given a brief which aimed at generating new forms of creative engagement that resonate and effectively communicate the personal research project to the public to generate learning. In addition, the Make it Public project has been developed in collaboration with the Design Museum in London with the intention to inform new form approaches of digital engagement.

In conclusion this paper has described how a project deploying public engagement as a method to develop qualitative relationships between Research and Innovation can inform an epistemological learning experience able to respond to the social transformations of the knowledge economy. With the goal of promoting multi-directional, interdisciplinary and negotiated experiences of learning the RCA MRes has explored what model of education could guide people in their upskilling journey. Through the KT model it has been evidenced how the human can play a key role in designing social transformations by leveraging the capacity to reflect, assess and enquire information to design dialogue and communication across people of different backgrounds and cultures. With education playing a twofolded role of providing the environment and the methods that enable a new experience of learning new skills can be shaped to construct an inclusive and sustainable future of work. This has been articulated 
through the description of the MRes Make it Public agenda which 7 projects produce outcomes that are indeed new forms of learning achieved through different media. By accounting the limitations given by its early stage of testing the MRes Make it Public has the ambition to be further developed in other contexts that would benefit from an interdisciplinary and multilateral experience and attitude towards learning by leveraging the role of the human in generating economic growth. Under these terms a creative and negotiated dialogue between industry and education can foster transformations that shape the skills necessary to develop inclusive human-to-human interactions with advanced technology and human led workplaces. The learning experience described in this paper has aimed to reframe innovation as a creative and collaborative learning process that values human intelligence; this is supported by a model of disseminated education which is designed upon the attitude of enquiring, criticising and assessing information across a lifetime through collaborative, diverse, creative and multidirectional dialogues.

\section{REFERENCES}

[1] Brassey. J., van Dam, N., Coates, K., Open interactive popup Seven essential elements of a lifelong-learning mind-set, in McKinsey \& Company, February 2019, https://www.mckinsey.com/business-functions/organization/our-insights/seven-essentialelements-of-a-lifelong-learning-mind-set [Accessed on 2020, 8 March]

[2] Cremin, T., \& Barnes, J., Creativity in the curriculum. Learning to teach in the primary school, 2015, 357-373.

https://www.researchgate.net/profile/Teresa_Cremin/publication/284039696_Creativity_in_the_c urriculum/links/56c2686c08ae44da37ff7a71/Creativity-in-the-curriculum.pdf [Accessed on 2020, 8 March]

[3] European Commission, Supporting growth and jobs - An agenda for the modernisation of Europe's higher education systems (COM (2011) 567 final, 20.09.2011), Brussels: European, (2011b) Commission. https://eur-lex.europa.eu/legal content/EN/TXT/PDF/?uri=CELEX:52011SC1063\&from=EN [Accessed on 2020, 8 March]

[4] European Commission, Report from the Commission to the Council, the European Parliament, the European Economic and Social Committee and the Committee of the Regions - Report on progress in quality assurance in higher education $\operatorname{COM(2009)} 487$ final, 21.9.2009 http://eurlex.europa.eu/LexUriServ/LexUriServ.do?uri=COM:2009:0487:FIN:EN:PDF in European Commission (2011b) [Accessed on 2020, 8 March]

[5] Fitzgerald, D., \& Callard, F., Entangling the medical humanities. Edinburgh University Press 2016.

[6] Fox, N. J. (2011). Boundary objects, social meanings and the success of new technologies. Sociology, 45(1), 70-85.

[7] Madgavkar, A., Manyika, J., Krishnan, M., Woetzel, J., Chui, M., Ellingrud, K., Yee, L., Hunt, V., Balabrishnan, S., The Future of Woman at Work. Transition in the Age of Automation, in Mckinsey\&Company, June 2019 https://www.mckinsey.com/featured-insights/genderequality/the-future-of-women-at-work-transitions-in-the-age-of-automation, [Accessed on 2020, $8 \mathrm{March}]$

[8] Nisbet, M. C., \& Markowitz, E. (2015). Public engagement research and major approaches. AAAS Centre for Public Engagement with Science and Technology Washington: Washington, DC, USA.

[9] OECD, 21st Century Learning and Teaching: Innovative Learning Environments, in OECD https://www.oecd.org/education/ceri/40805069.pdf [Accessed on 2020, 8 March]

[10] Schlegelmilch, B. B. (2020). Why Business Schools Need Radical Innovations: Drivers and Development Trajectories. Journal of Marketing Education, 0273475320922285.

[11] Unger, M., \& Polt, W, The knowledge triangle between research, education and innovation-a conceptual discussion. Форсайт, 2017, 11(2 (eng)). 